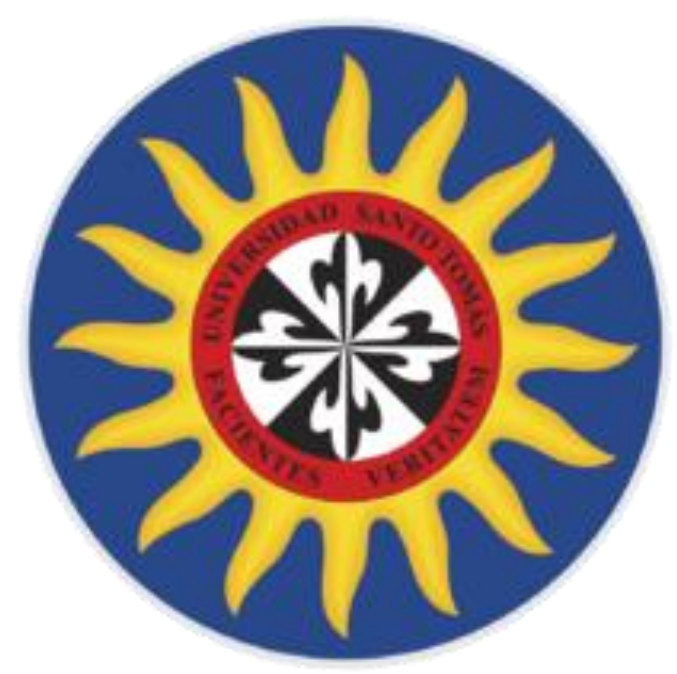

NORMA ALEXANDRA CUBILLOS ROSAS

UNIVERSIDAD SANTO TOMAS

FACULTAD DE CONTADURÍA PÚBLICA

VILLAVICENCIO

2018 


\section{TRABAJO SOCIAL EN LA UNIDAD DE PROYECCIÓN SOCIAL DE LA UNIVERSIDAD SANTO TOMÁS VILLAVICENCIO.}

NORMA ALEXANDRA CUBILLOS ROSAS

Informe de trabajo social presentado como requisito para optar al título de Contador Público

Asesor

Esp. HERNAN EDUARDO LASKAR ACERO

Especialización en gestión publica

UNIVERSIDAD SANTO TOMAS

FACULTAD DE CONTADURÍA PÚBLICA

VILLAVICENCIO

2018 
Autoridades Académicas

P JUAN UBALDO LÓPEZ SALAMANCA, O.P.

Rector General

\section{P MAURICIO ANTONIO CORTES GALLEGO, O.P.}

Vicerrector Académico General

P JOSÉ ARTURO RESTREPO RESTREPO, O.P.

Rector Sede Villavicencio

P FERNANDO CAJICÁ GAMBOA, O.P.

Vicerrector Académico Sede Villavicencio

Adm. JULIETH ANDREA SIERRA TOBÓN

Secretaria de División Sede Villavicencio

\section{Dr. JAVIER MAURICIO ÁLZATE TABARES}

Decano de la Facultad de Negocios Internacionales 


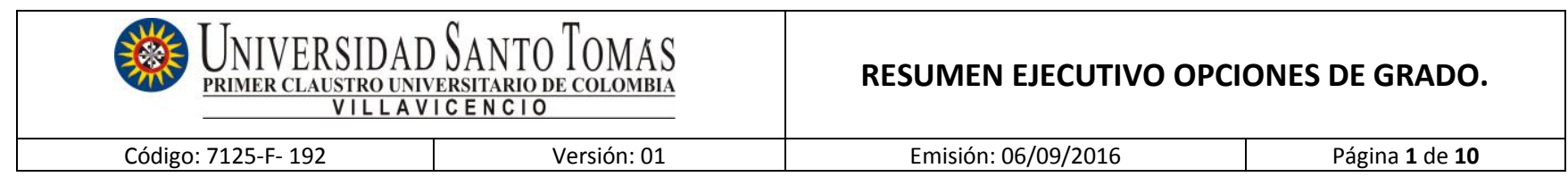

FACULTAD DE CONTADURÍA PÚBLICA

COORDINACIÓN DE OPCIONES DE GRADO.

\begin{tabular}{|l|l|}
\hline Tipo De Actividad & $\begin{array}{l}\text { Marque con una (X) } \\
\text { la opción de grado. }\end{array}$ \\
\hline Curso de Grado & \\
\hline Trabajo de Grado & \\
\hline Preparatorios & \\
\hline Promedio Ponderado & \\
\hline Trabajo Social & $\mathrm{X}$ \\
\hline
\end{tabular}

RESUMEN EJECUTIVO ACADÉMICO.

Proyecto o Actividad desarrollada objeto de informe manejada por el equipo:

Trabajo social apoyo Unidad de Proyección Social

Conformación del equipo de trabajo:

Docente Celia Cristina Cañas Olivo

Universidad (es) vinculada (s) en el desarrollo del proyecto o actividad:

Universidad Santo Tomás

Facultad (es) vinculada (s) en el desarrollo del proyecto o actividad:

Facultad de Contaduría Pública.

Empresa(s) vinculada (s) en el desarrollo del proyecto o actividad área(s) funcional(es) específica(s) de Trabajo:

Universidad Santo Tomás

Ciudad y fecha de realización del Informe:

Villavicencio, Meta, 05 de enero de 2018 


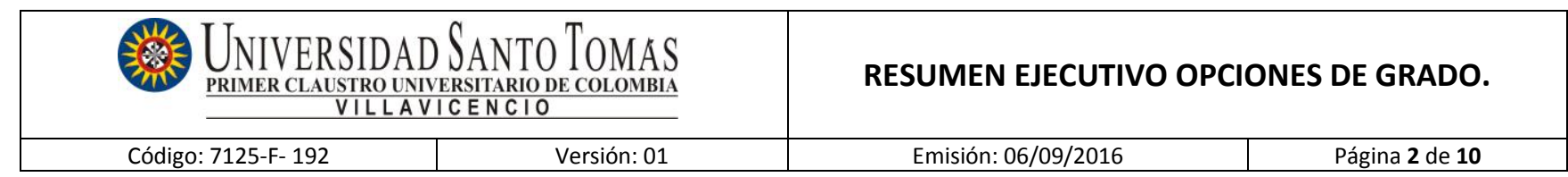

\section{INTRODUCCIÓN}

Este informe refleja las actividades realizadas desde la Unidad de Proyección Social, que fueron desarrolladas dentro de la Universidad Santo Tomás, demostrando el cumplimiento del plan de trabajo propuesto para tal fin, de igual forma contiene los resultados de cada labor realizada, y señala las funciones que se desarrollaron a lo largo de la duración de esta, describe la experiencia de esta práctica, recomendaciones y logros adquiridos durante la labor.

La práctica profesional es un espacio que permite al estudiante demostrar lo aprendido durante su carrera, es pasar de la teoría a la práctica del conocimiento adquirido, donde se asumen responsabilidades, y se enfrentan a diferentes ambientes, generando una similitud a lo que puede llegar a ser la vida laboral, es allí donde el pasante demuestra sus habilidades y destrezas en lo que tiene que ver con cumplimiento de tareas, comunicación, trabajo en equipo, entre otras.

\section{DESCRIPCIÓN}

\section{Objetivo General}

Apoyar los procesos que tiene a cargo la Unidad de Proyección Social de la Universidad Santo Tomás.

\section{Objetivos Específicos}

- Apoyar las funciones de Desarrollo Comunitario a cargo de la Unidad de Proyección Social, donde se realizaron creaciones de estadísticas como: estudio de impacto que tiene las clases dictadas en la Sede Loma Linda, analizando el número de personas que continúan aprovechando las clases brindadas y el número de personas que desiste de ellas.

- Cumplir con las funciones que sean asignadas por la docente Celia Cristina Cañas Olivo Líder de la Unidad de Proyección Social. Como la organización del archivo magnético y físico de la unidad a cargo de la docente, apoyando de igual forma las actividades interinstitucionales que se realizaron, juntos con los deberes ya descritos se suman la realización de actas debidas después de cada reunión y eventos realizados a cargo de la docente.

- Apoyar la oficina del consultorio empresarial, donde se realizaron las debidas labores para llevar a cabo reuniones con empresarios que les fuere de interés los servicios que se prestan desde la Facultad de Contaduría Pública. 


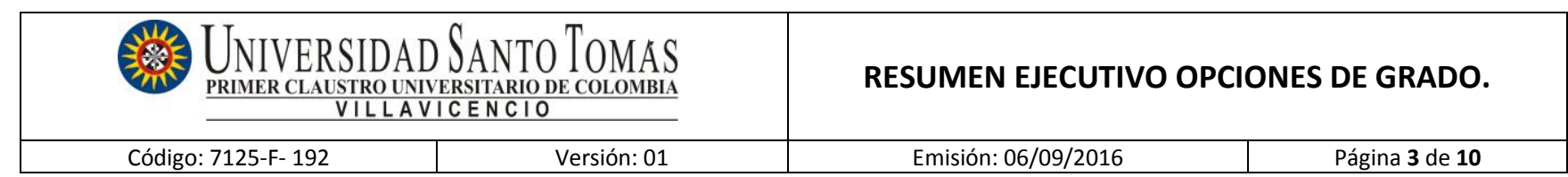

\section{METODOLOGÍA.}

La opción de grado fue realizada en la Universidad Santo Tomás, Sede Villavicencio como, apoyo a la Unidad de Proyección Social, el jefe inmediato fue la docente Celia Cristina Cañas Olivo, dentro de las funciones y responsabilidades del pasante se encontraban:

- Tabulación de encuestas de satisfacción y conocimiento realizadas a estudiantes beneficiarios de las clases en el Centro de Proyección Social de la Universidad Santo Tomás, Campus Loma Linda.

- Recopilación de informes ejecutivos de estudiantes graduados y estudiantes no graduados desde el año 2016-1 hasta el año 2018-1.

- Realización de actas de las reuniones lideradas por el Área de Proyección Social.

- Llamadas a empresarios para ofrecer servicios contables que brinda el Centro Empresarial de la Universidad Santo Tomás.

- Complementar información para la realización del documento maestro, datos de los estudiantes en práctica 2018-2, convenios que la Universidad ha tenido durante el semestre, eventos organizados por la Facultad de Contaduría Pública, entre otras.

- Apoyo en la creación del archivo de la docente, en el Área de Proyección Social.

- Apoyo en eventos que están a cargo de la docente Celia Cristina Cañas Olivo.

Para completar y alimentar cada una de las labores, se contó con el apoyo de estudiantes y administrativos de la Universidad, Diana Marcela Martínez Orjuela, secretaria de la Facultad de Contaduría Pública, fue apoyo para la recopilación y organización de información necesaria como:

- Cuadro maestro

- Base de datos de estudiantes graduados

- Base de datos de opciones de grado desde el año 2016-1 al año 2018-2

\section{LOGROS ALCANZADOS - RESULTADOS.}

Dentro de los logros obtenidos durante la práctica profesional se destacan los siguientes:

- Estudio de impacto de los cursos de contabilidad que se brindan en Loma Linda, este estudio se basó en: encuestas que se han realizado por semestre, a los beneficiarios de los diferentes cursos ofertados, el estudio se hizo con el fin de analizar y promediar quienes, y cuantos han permanecido en este programa, de igual forma se analizan los niveles de deserción. 


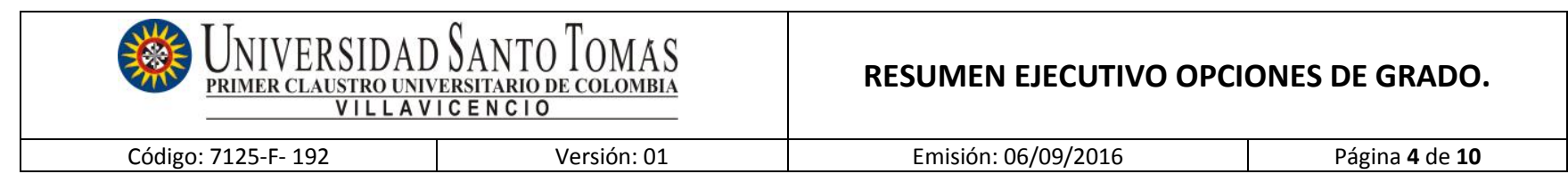

- Creación del archivo de la Unidad de Proyección Social, donde se refleja cada labor realizada desde esta área, como eventos, reuniones, capacitaciones. Durante el tiempo de práctica se realizaron dos eventos que fueron organizados por la Junta Central de Contadores y por el Consejo Técnico de Contaduría, se brindó una capacitación a empresas palmicultoras de la región, sobre la NIC 41 Activos Biológicos. Cada evidencia fue consignada dentro de este archivo.

- Llamadas realizadas a empresarios para dar a conocer los servicios del Consultorio Empresarial de la Facultad de Contaduría Pública, con el fin de concretar citas y determinar si los servicios ofrecidos son de su interés.

\section{CONCLUSIONES.}

- El estudio de impacto reflejó la ausencia y permanencia de las personas que toman las clases de contabilidad brindadas en la sede Loma Linda de la Universidad Santo Tomás, donde brevemente se cuenta el por qué desisten los estudiantes a los cursos, al finalizar este estudio fue expuesto como ponencia en la Universidad de los Llanos, mostrando cada uno de los resultados y el impacto que ha tenido esta labor en el desarrollo de las comunidades beneficiadas.

- Finalizado el tiempo de la práctica se hace entrega al Decano de la Facultad de Contaduría Publica el archivo digital y físico de la Unidad de Proyección Social, donde se evidencia cada uno de los eventos, actas, listas de asistencias, evidencias fotográficas y demás documentos que refleja las labores realizadas durante el semestre 2018-2, eventos que estaban a cargo de la Unidad de Proyección Social y de la docente Celia Cristina Cañas Olivo, de igual forma las reuniones que se tuvieron con los empresarios a quienes se les daba a conocer los servicios que ofrece el Centro Empresarial de la Universidad Santo Tomás.

- En la oficina del Centro Empresarial de la Universidad se realizaban llamadas para contactar empresarios que se interesaran por los servicios contables ofrecidos por la misma, en otros momentos en la oficina también se dio lugar a la organización de la capacitación que fue brindada a los empresarios palmicultores donde se realizaron llamadas con el fin de concretar y 


\begin{tabular}{|c|c|}
\hline 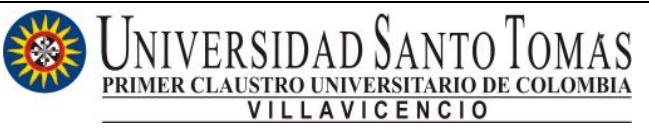 & RESUMEN EJECUTIVO OPCIONES DE GRADO. \\
\hline Código: 7125-F- 192 & Emisión: 06/09/2016 \\
\hline
\end{tabular}

formalizar las citas para que fuere participes de la capacitación. Este lugar fue un espacio provechoso ya que permitió y facilitó el desarrollo de muchas de las labores a cargo, pues contaba con teléfono y computador a disposición para el desarrollo de las actividades.

\section{RECOMENDACIONES.}

La opción de grado se realizó dentro de la Universidad Santo Tomás, lo que permitió observar que no existe una adecuada organización de los archivos físicos de eventos o reuniones porque no cuentan con una información completa, esto en algunas ocasiones dificultaba el progreso de las labores ya que la información faltante era necesaria para el desarrollo de las actividades a cargo de la pasante.

\section{REFERENTES BIBLIOGRÁFICOS, VIDEOS, WEB Y CON EXPERTOS}

\section{ANEXOS}

\section{Actualización Normativa en Contabilidad y Aseguramiento}

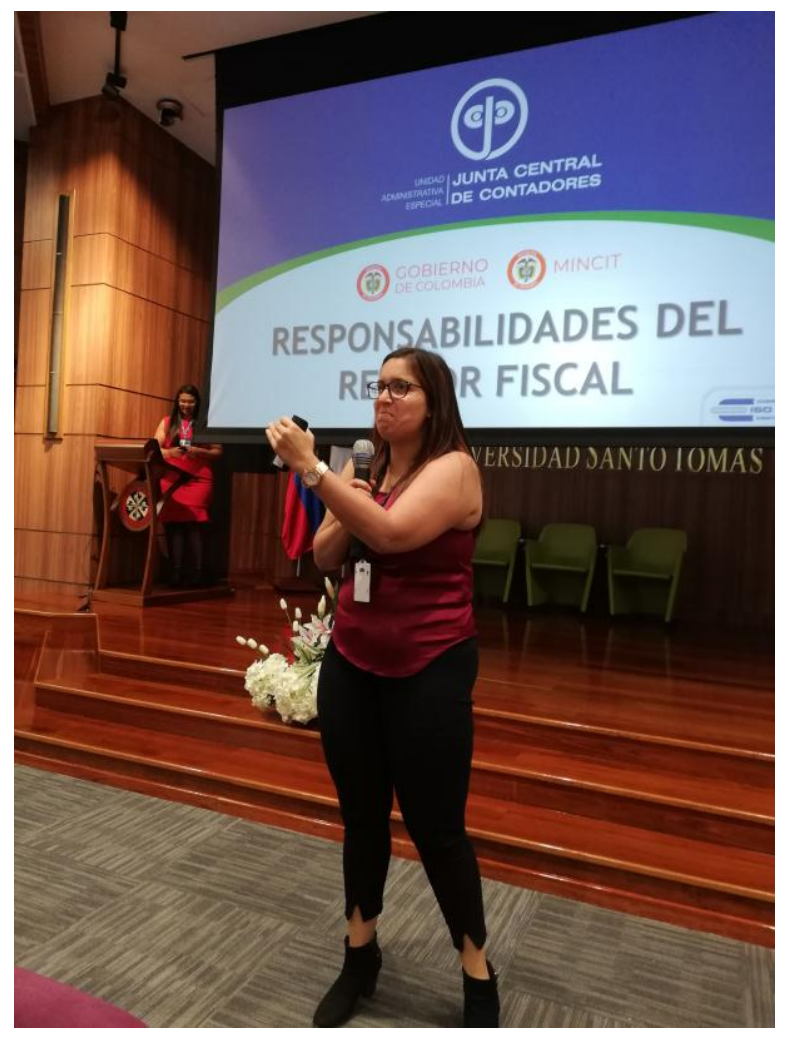

Fotografía 1 Actualización Normativa en Contabilidad y Aseguramiento.

Fuente fotografía tomada por Norma Cubillos, Villavicencio, Meta (2018) 


\begin{tabular}{|c|c|c|c|}
\hline 潘 UNIV NIVER & TOMAS & \multicolumn{2}{|c|}{ RESUMEN EJECUTIVO OPCIONES DE GRADO. } \\
\hline Código: 7125-F- 192 & Versión: 01 & Emisión: 06/09/2016 & Página 6 de 10 \\
\hline
\end{tabular}

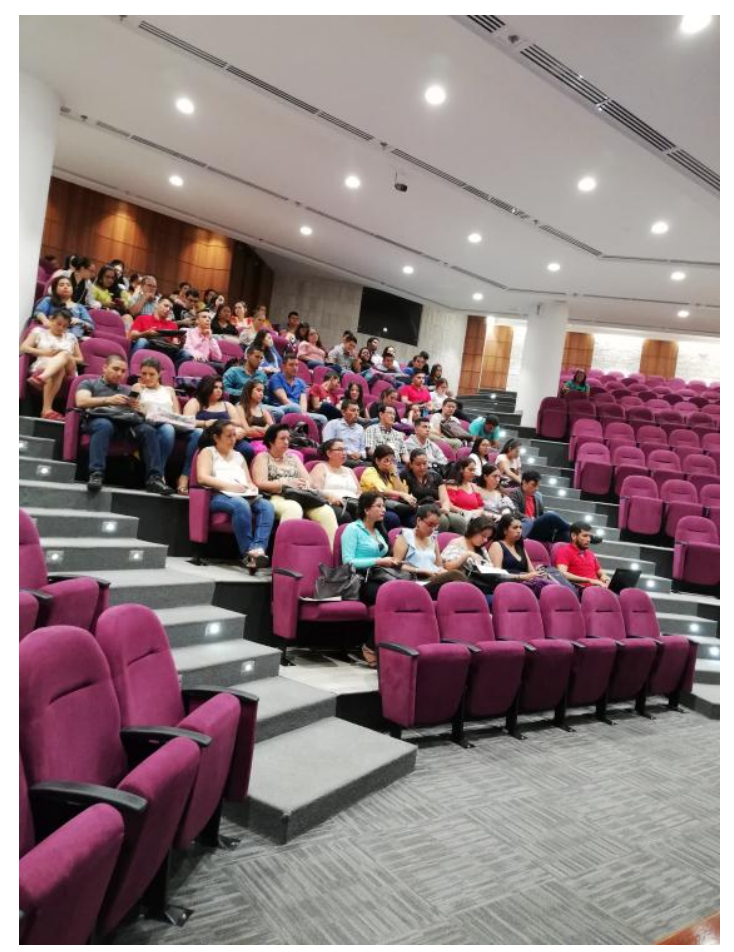

Fotografía 2 Actualización Normativa en Contabilidad y Aseguramiento.

Fuente fotografía tomada por Norma Cubillos, Villavicencio, Meta (2018)

Tratamiento Contable de Activos Biológicos en la Actividad Palmicultora. NIC 41

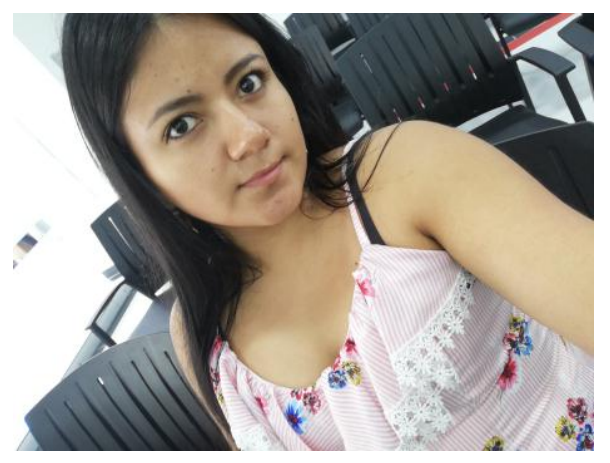

Fotografía 3 Capacitación sector Palmicultor

Fuente fotografía tomada por Norma Cubillos, Villavicencio, Meta (2018) 


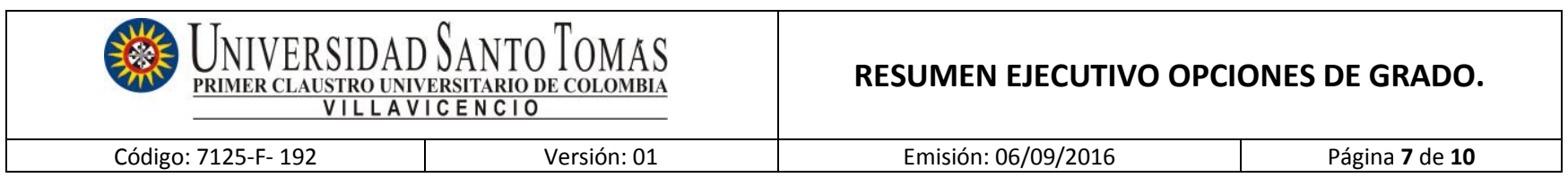

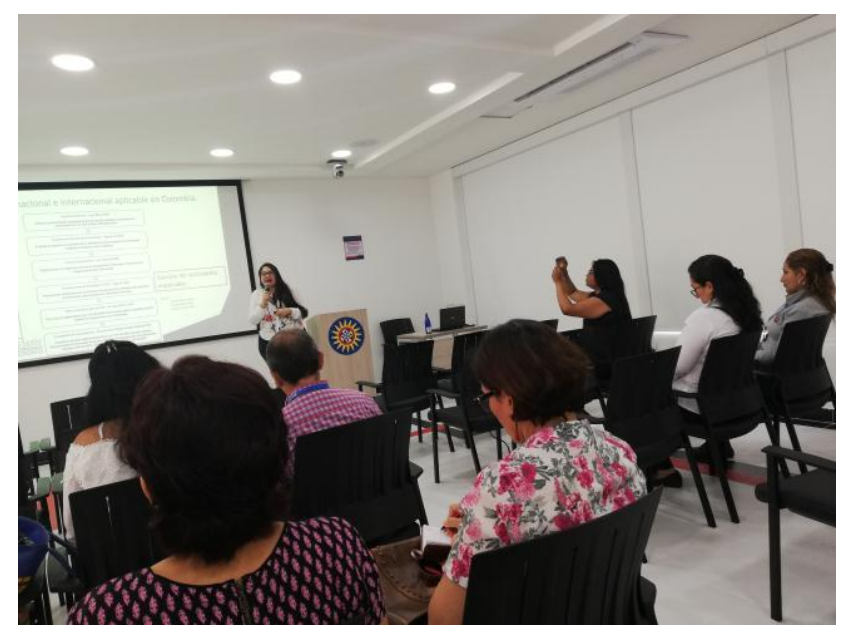

Fotografía 4 Capacitación sector Palmicultor

Fuente fotografía tomada por Norma Cubillos, Villavicencio, Meta (2018)

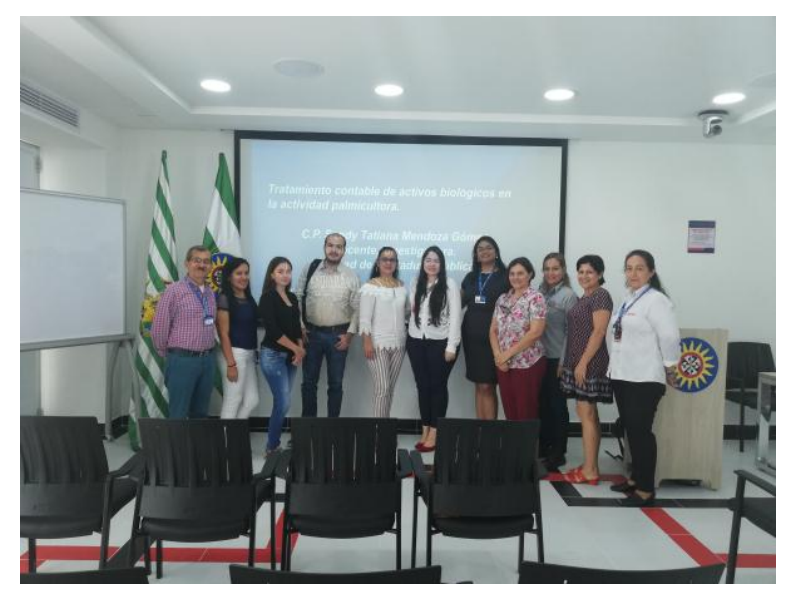

Fotografía 5 Capacitación sector Palmicultor

Fuente fotografía tomada por Norma Cubillos, Villavicencio, Meta (2018) 


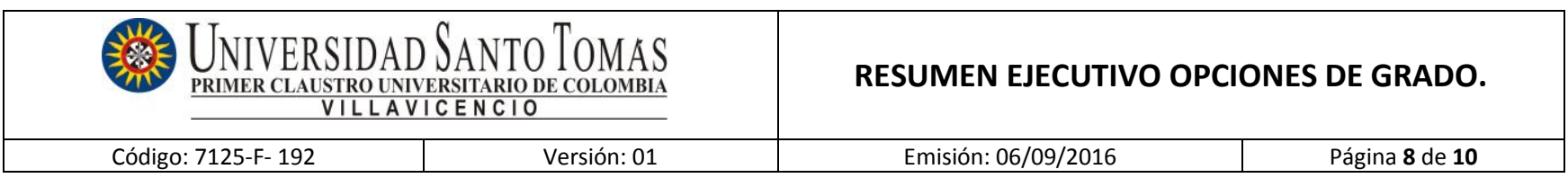

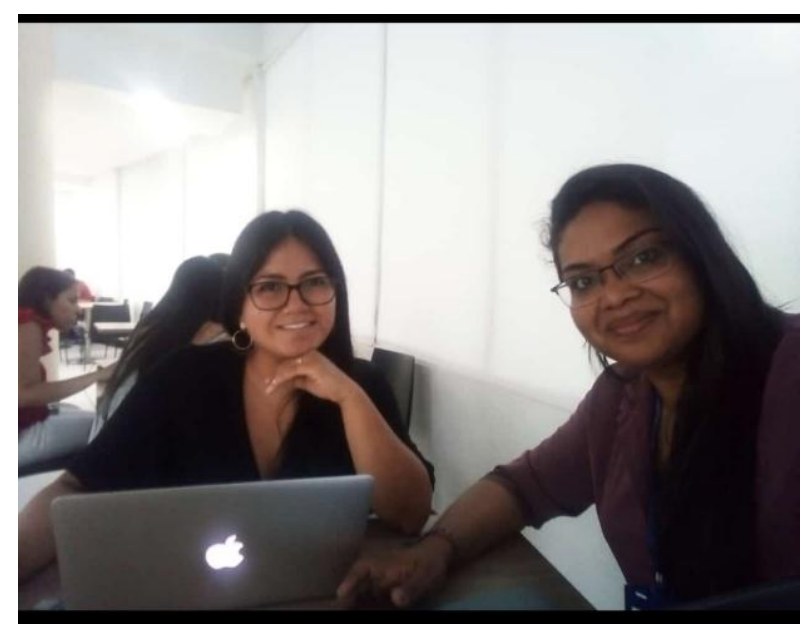

Fotografía 6 Revisión del archivo

Fuente fotografía tomada por Celia Cañas, Villavicencio, Meta (2018)

Entregado en a los días del mes de del año

\section{Quien entrega:}

\section{Nombre y Documento}

Norma Alexandra Cubillos Rosas

1.121.939.942

Calidad: Estudiante

TABLA DE VALORACIÓN

\begin{tabular}{|l|l|l|l|l|}
\hline Aspecto & \multicolumn{2}{|l|}{ Nivel de cumplimiento } \\
\cline { 2 - 5 } & Muy Alto & Alto & Medio & Bajo \\
\hline $\begin{array}{l}\text { Contiene todos los elementos } \\
\text { establecidos en el documento } \\
\text { "Resumen Ejecutivo Académico". }\end{array}$ & & & & \\
\hline $\begin{array}{l}\text { Se hace explícito los resultados de } \\
\text { manera clara y concreta. }\end{array}$ & & & & \\
\hline
\end{tabular}




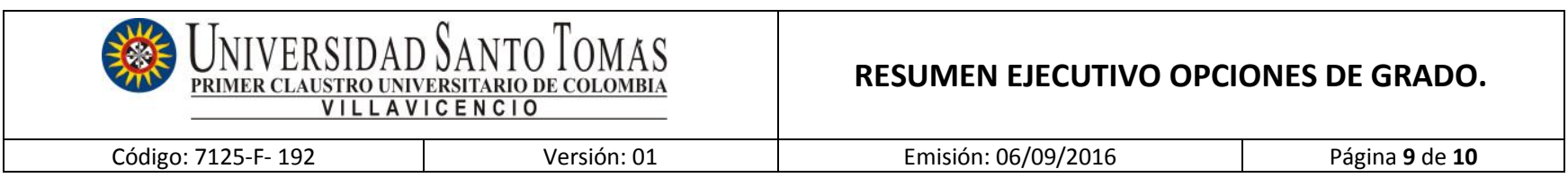

\begin{tabular}{|l|l|l|l|l|}
\hline $\begin{array}{l}\text { Hay coherencia en la construcción del } \\
\text { documento. }\end{array}$ & & & \\
\hline $\begin{array}{l}\text { Evidencia revisión de fuentes de } \\
\text { información. }\end{array}$ & & & & \\
\hline $\begin{array}{l}\text { Cumplimiento de condiciones de } \\
\text { entrega. }\end{array}$ & & & \\
\hline
\end{tabular}

Se considera aceptado el documento cuando cumpla con todos los aspectos en su totalidad.

Validado en

a los

días del mes de del año

Quien Valora el documento:

Firma

Nombre:

Documento:

Calidad: Docente Coordinador del proceso.

Firma

Nombre:

Documento:

Calidad: Coordinador Opciones de Grado.

QUIEN RECIBE EN LA FACULTAD PARA ARCHIVO:

Firma 


\begin{tabular}{|c|c|}
\hline 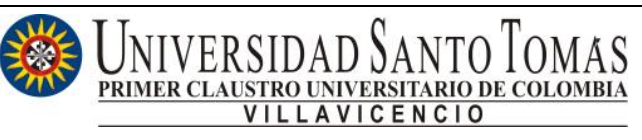 & RESUMEN EJECUTIVO OPCIONES DE GRADO. \\
\hline Código: 7125-F- 192 & Emisión: 06/09/2016 \\
\hline
\end{tabular}

\section{Nombre:}

Documento:

Cargo:

Fecha: 